\title{
Koebner Phenomenon and Mycosis Fungoides
}

\author{
Eve Lebas Florence Libon Arjen F. Nikkels \\ Department of Dermatology, CHU Sart Tilman, University of Liège, Liège, Belgium
}

\section{Key Words}

Mycosis fungoides · Primary cutaneous T-cell/NK-cell lymphoma $\cdot$ Koebnerization ·

Isomorphic effect · Traumatism

\begin{abstract}
Mycosis fungoides (MF) is the most frequent type of primary cutaneous T-cell/NK-cell lymphoma. The Koebner phenomenon is defined as the appearance of cutaneous lesions on previously noninvolved skin following trauma and is observed in a series of cutaneous diseases including psoriasis, lichen planus, viral warts, molluscum contagiosum, etc. In this case report, 3 patients with longstanding MF are presented, the 1st with the appearance of a circumscribed early-stage type MF lesion rapidly following a surgical excision of an infundibular cyst, the 2nd with the appearance of a unique unilateral palmar tumoral MF lesion at the pressure site of a crutch, and the 3rd presented localized MF early stage lesions at the friction site of a belt. This report suggests that some MF patients may experience Koebner phenomenon-induced MF lesions and that MF should be added to the long list of skin diseases potentially exhibiting the Koebner phenomenon.

(c) 2015 S. Karger AG, Basel
\end{abstract}

\section{Introduction}

Koebner described the isomorphic phenomenon in 1872 by reporting the appearance of psoriasis on previously noninvolved skin after skin trauma [1-3]. Besides psoriasis, there is an ever-expanding array of dermatoses that can arise following cutaneous injury. Currently, 4 different circumstances of isomorphic reactions are distinguished [4]. The term true koebnerization describes the reproducible Koebner phenomenon induced by an external injury, as observed in psoriasis and lichen planus. The designation pseudo-koebnerization is used for cutaneous infections that can disseminate infectious agents, including warts or molluscum contagiosum. Occasional koebnerization is reserved when some criteria for the 
Lebas et al.: Koebner Phenomenon and Mycosis Fungoides

Koebner response are encountered but not always reproducible, like in Darier disease, erythema multiforme, granuloma annulare and Behçet disease. The last category represents the questionable trauma-induced processes, including all the cutaneous disorders reported as case reports that evoke a link to injury, such as pemphigus vulgaris, discoid lupus erythematosus, lupus erythematosus and eruptive xanthoma.

Mycosis fungoides (MF) is the most often encountered type of primary cutaneous Tcell/NK-cell lymphoma (pCTNKCL) [5]. The precise origin of pCTNKCL still remains enigmatic, although some hypotheses are proposed, such as chronic exposure to topical chemical agents [6] or professional exposure to chemicals [7]. Other risk factors for MF are longstanding psoriasis and urticaria [8], but atopic dermatitis and viral infections seem not to represent risk factors.

We present 3 patients with various stages of MF lesions appearing on previously noninvolved skin after cutaneous trauma.

\section{Case Reports}

\section{Patient 1}

A 69-year-old patient with a 4-year history of MF presented the rapid occurrence of a well-circumscribed infiltrated plaque about 2 weeks after the surgical excision of an infundibular cyst in the right retroauricular region (fig. 1). At that moment, the patient was on a low-dose maintenance interferon therapy (Intron ${ }^{\circledR} \mathrm{A}$; Shering-Plough, $2 \times 3.10^{6} \mathrm{IU}$ per week) and presented a clinical stage of T1N0M0B0. Clinical and biological treatment tolerance was excellent. A 3-mm punch biopsy was performed under local anesthesia, and histological analysis confirmed early-stage MF with a high number of atypical lymphocytes infiltrating the epidermis. Immunohistochemical staining showed positive CD4 staining of the infiltrating lymphocytes. A monoclonal T-cell receptor rearrangement was evidenced in the sample. As the patient had a stable disease, the dosing of interferon was not increased, and the plaque was treated with very potent topical steroids (clobetasol propionate $0.05 \mathrm{cream}$, $1 \times /$ day, for 4 weeks). The lesion resolved progressively. There was no history of recently introduced new agents. The patient had never experienced this Koebner phenomenon previously. However, 3 months later, the MF lesion reappeared exactly at the same place with the same clinical aspect. The lesion was again successfully treated with topical corticosteroids.

\section{Patient 2}

A 51-year-old woman presented with tumoral MF (T3N0M0B0). After 2 months of highdose interferon (IntronA, $3 \times 9.10^{6}$ UI per week), the tumoral lesions slunk to slightly infiltrated plaques. Biological and clinical tolerance was unremarkable. The interferon dose was tapered over 4 weeks, and methotrexate maintenance therapy was initiated $10 \mathrm{mg}$ per week). She remained stable for several months. After a fall, she used a crutch with her right arm due to low back pain. About 1 month after her fall, she presented a tumoral MF-like lesion of the palmar aspect of her right hand. The lesion developed precisely at the pressure site of her crutch (fig. 2). She had not experienced any other local traumatism. No other T3type lesions occurred on other body sites. No new drugs were initiated. A 3-mm punch biopsy was performed, and histology confirmed tumoral MF. The patient refused to return to interferon therapy as the lesion was not bothering her. Topical potent steroids (clobetasol propionate 0.05 cream, $1 \times /$ day) and weekly intralesional infiltrations of corticosteroids (triamcinolone acetonide $10 \mathrm{mg} / \mathrm{ml}$, Albicort ${ }^{\circledR}$; Sanofi-Aventis) were administered with an 
Lebas et al.: Koebner Phenomenon and Mycosis Fungoides

acceptable clinical regression after 5 weeks. As her condition improved steadily, she refrained from using her crutch, and no recurrence was observed on her hand.

Patient 3

A 57-year-old man presented a longstanding history of extensive cutaneous MF (T2N0M0B0). Besides topical steroids, he had previously received several courses of UVB and rePUVA treatments with good clinical tolerance and results. After a complete remission period of 6 months, he presented again for recurring MF, especially localized at his belt. At this moment, the staging was T1N0M0B0. Clinical examination revealed some small and slightly infiltrated and isolated MF plaques. Curiously, limited at a friction zone on his right hip due to his belt, he presented serpiginous infiltrated and erythematous lesions (fig. 3). He had never observed this phenomenon previously. He had not received a local traumatism. A 3-mm punch biopsy was taken to exclude psoriasis induced by the Koebner phenomenon. However, histology confirmed early-stage MF. As the patient did not present at that time more than 10 skin lesions, it was decided to treat him by very potent topical corticosteroids (clobetasol propionate 0.05 cream, $1 \times /$ day). After approximately 4 weeks, the lesions were as good as resolved. He presented no more recurrences at that site despite continuing wearing his belt.

\section{Discussion}

The vast majority of cutaneous diseases displaying the Koebner phenomenon are of inflammatory nature. The 3 cases described here are probably best classified in the third category of occasional Koebner phenomenon [4]. In the psoriasis literature, it has been described that scar sites are particularly prone to the Koebner effect, related to the changes in local vascularization and neoangiogenesis [4]. Psoriasis disease severity seems not to present a risk factor, but stress factors do. The precise pathogenic understanding of Koebner phenomenon remains enigmatic, but probably a whole array of stress proteins, cytokines, neuromediators including nerve growth factor, growth factors like basic fibroblast growth factor and adhesion molecules are certainly intervening [3]. It is hypothesized that the atypical MF cells usually displaying a CD3+, CD4+, CD8- and CD45R0+ phenotype, and a Th2 cytokine production profile, find a favorable environment for disease induction, activation or reactivation [5].

A recent report described 4 patients developing MF on previously traumatized skin sites [9]. All these patients were male and presented a median time of 10 years between the cutaneous traumatism and the diagnosis of MF. The transdermal estradiol patch-restricted MF [10] and solvent-induced tumor d'emblée MF [6] are probably related to the chronic exposure to chemical agents. Although traumatic in origin, the very long time interval between traumatism and the occurrence of MF does not correspond to the definition of a Koebner effect.

In sum, these case presentations suggest that MF early stage and tumoral stage lesions may point to the Koebner phenomenon. MF should be added to the list of dermatoses exhibiting the Koebner phenomenon. 
Lebas et al.: Koebner Phenomenon and Mycosis Fungoides

\section{Statement of Ethics}

This study was performed in accordance with the ethics standards of the University Hospital Committee on Institutional Human Experimentation and with the Declaration of Helsinki of 1975, as amended in 1983. The aims of the study were explained to all the patients, and all signed an informed consent.

\section{Disclosure Statement}

The authors declare no conflicts of interests. No funding was obtained for this work.

\section{References}

1 Kuner N, Hartschuh W, Khan-Durani B: Heinrich Köbner and the 'isomorphic phenomenon'. History and review of the literature. Hautarzt 2003;54:274-278.

$\longrightarrow 2$ Weiss G, Shemer A, Trau H: The Koebner phenomenon: review of the literature. J Eur Acad Dermatol Venereol 2002;16:241-248.

3 Sagi L, Trau H: The Koebner phenomenon. Clin Dermatol 2011;29:231-236.

4 Boyd AS, Neldner KH: The isomorphic response of Koebner. Int I Dermatol 1990;29:401-410

5 Wong HK, Mishra A, Hake T, Porcu P: Evolving insights in the pathogenesis and therapy of cutaneous T-cell lymphoma (mycosis fungoides and Sezary syndrome). Br J Haematol 2011;155:150-166.

-6 Nikkels AF, Quatresooz P, Delvenne P, Balsat A, Piérard GE: Mycosis fungoides progression and chronic solvent exposure. Dermatology 2004;208:171-173.

7 Wohl Y, Tur E: Environmental risk factors for mycosis fungoides. Curr Probl Dermatol 2007;35:52-64.

-8 Morales MM, Olsen J, Johansen P, et al: Viral infection, atopy and mycosis fungoides: a European multicentre case-control study. Eur J Cancer 2003;39:511-516.

19 Paul LJ, Duvic M: Mycosis fungoides following skin trauma. J Am Acad Dermatol 2012;67:e148.

10 Alloo A, Desimone JA, Kupper TS: Mycosis fungoides presenting at the site of a transdermal estradiol patch. J Am Acad Dermatol 2012;67:e207-e208.

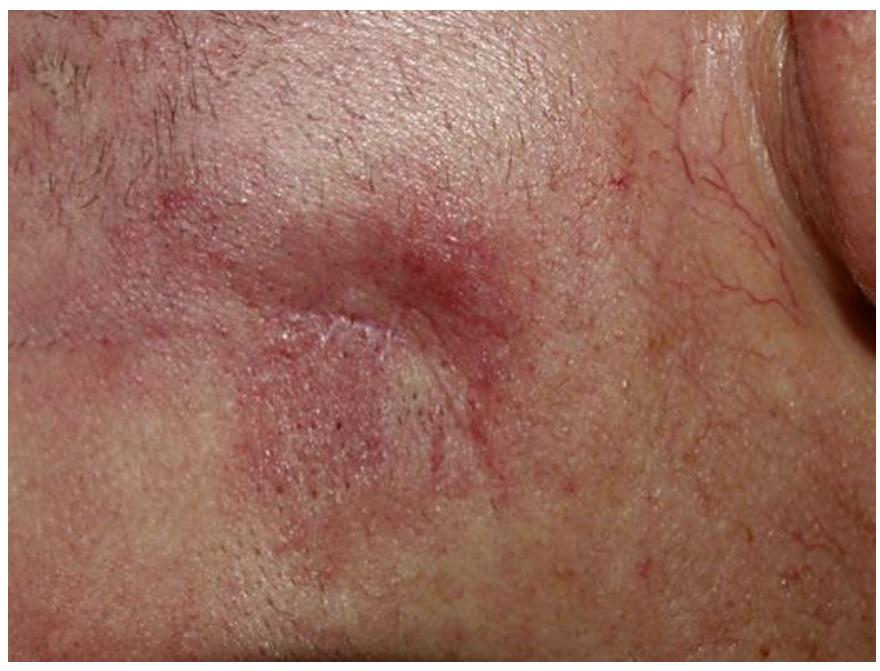

Fig. 1. Early-stage MF surrounding a recent surgical scar. 


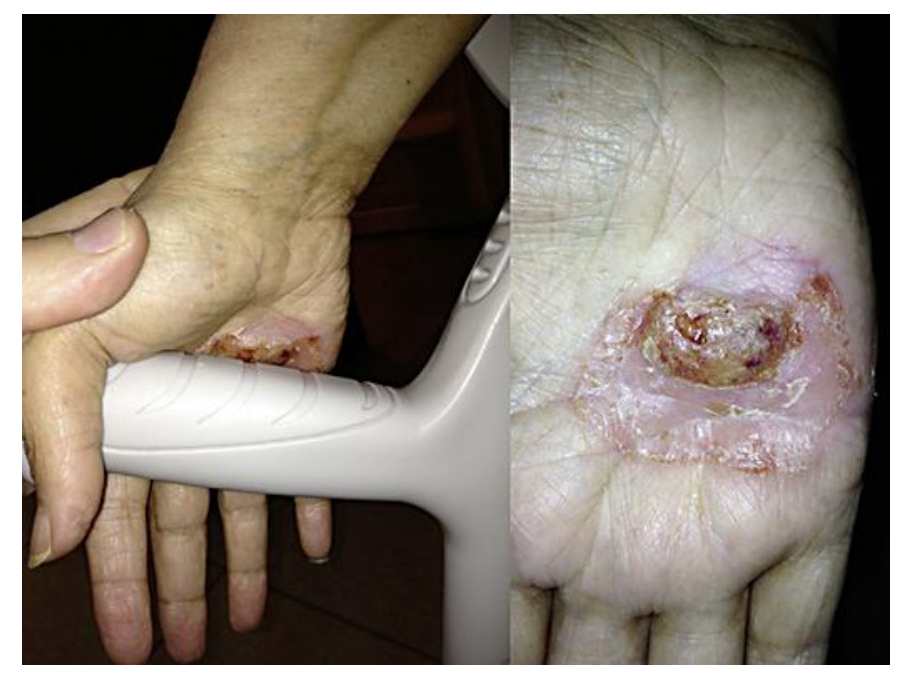

Fig. 2. Palmar tumoral MF at the pressure site of a crutch.

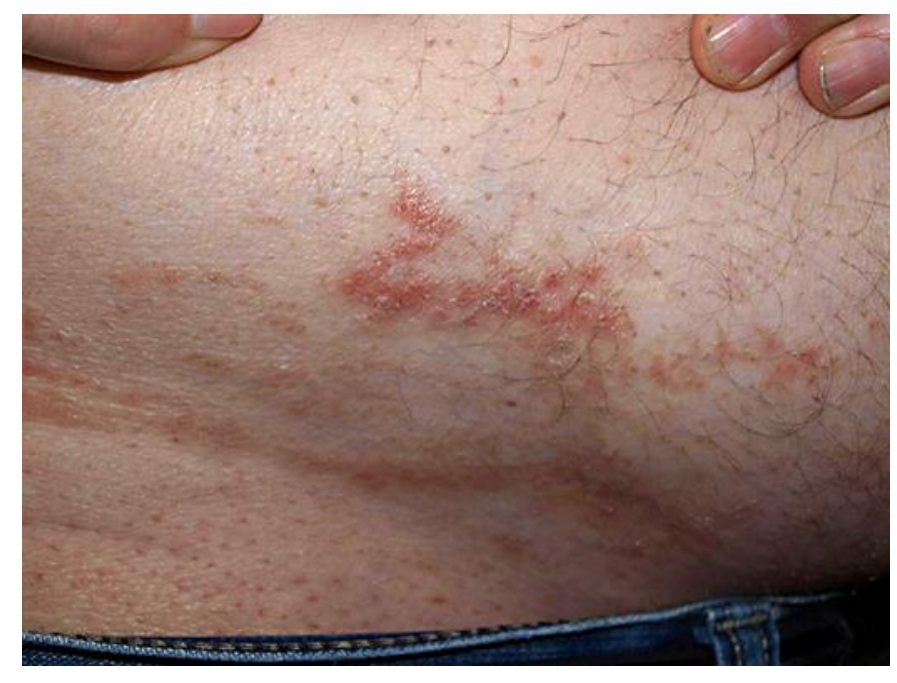

Fig. 3. Early-stage MF localized at the friction site of a belt. 